\title{
Phillygenin inhibits the inflammation and apoptosis of pulmonary epithelial cells by activating PPAR $\gamma$ signaling via downregulation of MMP8
}

\author{
YUFENG LIN ${ }^{1 *}$ and PENG YANG ${ }^{2 *}$ \\ ${ }^{1}$ Department of Pediatrics, Gaolangang Hospital of Zhuhai People's Hospital, Zhuhai, Guangdong 519050; \\ ${ }^{2}$ Department of PICU, The Second Affiliated Hospital of Shandong First Medical University, \\ Tai'an, Shandong 271000, P.R. China
}

Received December 17, 2020; Accepted March 10, 2021

DOI: $10.3892 / \mathrm{mmr} .2021 .12415$

\begin{abstract}
Acute lung injury (ALI) is often responsible for the high morbidity of critically ill patients. The present study aimed to investigate whether phillygenin (PHI) can inhibit inflammation and apoptosis of pulmonary epithelial cells by activating peroxisome proliferator-activated receptor $\gamma$ (PPAR $\gamma$ ) signaling. The in vitro model of ALI was established using lipopolysaccharide (LPS) and PHI was used to treat the LPS-induced cells. Cell viability was assessed using the MTT assay and the concentration levels of the inflammatory factors were detected by ELISA. Western blotting and reverse transcription-quantitative PCR were conducted to measure the expression levels of the inflammation- and apoptosis-associated proteins. The MMP8-overexpression plasmid was transfected into LPS-induced cells, which were treated with PHI treatment and the expression levels of PPAR $\gamma$ were detected via western blotting. PHI treatment suppressed the induction of inflammation and apoptosis of LPS-induced BEAS-2B cells. Furthermore, the expression levels of MMP8 in BEAS-2B cells induced by LPS were decreased following PHI treatment. Following transfection of the MMP8 overexpression plasmid into the LPS-induced BEAS-2B cells and subsequent treatment of these cells with PHI, the expression levels of PPAR $\gamma$ were decreased. In conclusion, it was shown that PHI inhibited the inflammation and apoptosis of pulmonary epithelial cells by activating PPAR $\gamma$ signaling via downregulating MMP8. These data may provide valuable information for future studies exploring the therapeutic effects of PHI for ALI.
\end{abstract}

Correspondence to: Dr Yufeng Lin, Department of Pediatrics, Gaolangang Hospital of Zhuhai People's Hospital, 13 Nangang Road, Jinwan, Zhuhai, Guangdong 519050, P.R. China

E-mail: linyufenglyf@163.com

${ }^{*}$ Contributed equally

Key words: phillygenin, peroxisome proliferator-activated receptor $\gamma$, MMP8, inflammation, apoptosis, pulmonary epithelial cells

\section{Introduction}

The lungs are one of the most vulnerable organs in the human body, and alveolar macrophages are the major inflammatory cells involved in the maintenance of the lung's defense against foreign pathogens (1). Phagocytosis caused by these macrophages and the accompanied release of cytokines constitute the host cellular defense (2). Acute lung injury (ALI) is responsible for the high morbidity of critically ill patients (3). By damaging the alveolar epithelium, ALI can trigger the inflammatory response in the defensive system of the lung (4). Despite the complex mechanism that contributes to the occurrence of ALI, the damage to the epithelial cells can speed up the development of this disease $(5,6)$. To date, specific pharmacological drugs have not been used in clinical practice for the treatment of ALI (7). Therefore, the severity of ALI and the potential targets or drugs that can treat this disease should be examined.

Phillygenin (PHI; 4-[(3S,3aR,6R,6aR)-6(3,4-dimethox yphenyl)-1,3,3a,4,6,6a-hexahydrofuro[3,4-c] furan-3yl]-2methoxyphenol) (Fig. 1A) is a lignan component extracted from Forsythiae Fructus (8), and it has been reported to exert anti-inflammatory effects (9). The Toll-like receptor (TLR)4/MyD88/NF- $\mathrm{B}$ signaling pathway, which can be activated by PHI, can inhibit lipopolysaccharide (LPS)-induced inflammation and further alleviate liver fibrosis (8). Furthermore, PHI has been reported to have significant antitumor effects in human esophageal and pancreatic cancer types $(10,11)$.

A search of the SwissTargetPrediction website suggested that PHI can bind to and regulate the expression of MMP8. Previous studies have indicated that MMP8 and MMP9 expression levels are elevated in pediatric patients with ALI $(11,12)$. Decreased MMP8 activity and aberrantly increased MMP9 activity were noted in patients with ALI with prolonged disease progression (13). MMP8 serves an important role in inflammation and degradation of tight junction proteins (14). Increased expression of MMP8 is associated with the early inflammation stage of spinal cord injury (SCI) and addition of a specific MMP8 inhibitor can markedly alleviate the inflammatory response and cellular damage of SCI (14). The 
use of MMP8 inhibitors on astrocytes can suppress the levels of inducible nitric oxide synthase, TNF- $\alpha$, IL-1 $\beta$, IL-6 and TLR2, and is accompanied by the activation of peroxisome proliferator-activated receptor $\gamma(\operatorname{PPAR} \gamma)$ and the inhibition of NF- $\kappa \mathrm{B}$ activity, which is induced by lipoteichoic acid (15). The notable therapeutic potential of curcumin in the treatment of neonatal ALI is mediated by activating PPAR $\gamma /$ heme oxygenase-1 signaling in an animal model (16). This finding provided evidence that activation of PPAR $\gamma$ may be a novel therapeutic strategy for the treatment of ALI. Therefore, the present study aimed to investigate whether PHI can inhibit the induction of inflammation and apoptosis of pulmonary epithelial cells by activating PPAR $\gamma$ signaling.

\section{Materials and methods}

Sample collection. The clinical study protocol was approved by Gaolangang Hospital of Zhuhai People's Hospital (approval no. 2020-012; Zhuhai, China) between 2019/09 and 2020/02. Signed informed consent forms were obtained from the children's family for the collection and use of the specimens. Briefly, patients who were admitted to the pediatric intensive care unit of Gaolangang Hospital of Zhuhai People's Hospital were enrolled. Inclusion criteria were pediatric patients [age, 2-10 years; 28 (46.7\%) females and 32 (53.3\%) males] who were intubated and mechanically ventilated with a ratio of partial pressure of arterial oxygen $\left(\mathrm{PaO}_{2}\right)$ to the fraction of inspired oxygen $\left(\mathrm{FiO}_{2}\right)$ of $\leq 300$ (adjusted to 253 in Salt Lake City due to altitude), bilateral pulmonary infiltrates and no clinical evidence of left atrial hypertension. Patients were excluded if they were $<2$ years of age; had respiratory failure from cardiac disease; had hypoxemia without bilateral infiltrates; had received a bone marrow or lung transplant; were supported on extracorporeal membrane oxygenation; had a non-pulmonary condition that could be exacerbated by the prone position; had participated in other clinical trials within the preceding 30 days; or if there was a decision to limit life support. Blood samples were collected within the first $24 \mathrm{~h}$ of diagnosis. The serum samples of pediatric patients with ALI $(n=30)$ and normal subjects $(n=30)$ were collected for further analysis following submission of the informed consent form.

Cell lines and treatment. The pulmonary epithelial cell line (BEAS-2B) was obtained from the American Type Culture Collection. The cells were maintained in RPMI-1640 medium (Thermo Fisher Scientific, Inc.) containing 10\% FBS (Thermo Fisher Scientific, Inc.) in an incubator (Thermo Fisher Scientific, Inc.) at $37^{\circ} \mathrm{C}$ with $5 \% \mathrm{CO}_{2}$. LPS was purchased from Sigma-Aldrich; Merck KGaA. For LPS induction, BEAS-2B cells were plated into 6-well plates and cultured for $48 \mathrm{~h}$. LPS (100 $\mathrm{ng} / \mathrm{ml})$ or saline solution was added and the cells were incubated for $24 \mathrm{~h}$ to induce inflammatory response and apoptosis. PHI (purity 99\%) was purchased from Chroma-Biotechnology Co., Ltd. (https://chroma-biotech. company.lookchem.cn/). PHI was used at the concentrations of $12.5,25$ and $50 \mu \mathrm{g} / \mathrm{ml}$. This compound was incubated with the cells $24 \mathrm{~h}$ following LPS induction (8).

Cell transfection. The MMP8-overexpression plasmid vector (pcDNA3.1-MMP8) and empty vector (pcDNA3.1-NC) plasmid were purchased from Shanghai GenePharma Co., Ltd. In accordance with the manufacturer's instructions, following LPS treatment, $1 \mu \mathrm{g}$ plasmids were transfected into BEAS-2B cells using Lipofectamine ${ }^{\circledR} 3000$ reagent (Invitrogen; Thermo Fisher Scientific, Inc.). Following incubation for $6 \mathrm{~h}$, the medium was replaced with RPMI-1640 medium with 10\% FBS (Thermo Fisher Scientific, Inc). After $48 \mathrm{~h}$ of incubation, the cells were collected and the transfection efficiency was analyzed by reverse transcription-quantitative PCR (RT-qPCR).

MTT assay. Cells were seeded in 96-well plates at a density of 3,000/well and were subsequently treated by different concentrations of PHI for $24 \mathrm{~h}$. A total of $10 \mu \mathrm{l}$ MTT solution was added to the medium and the cells were cultured for an additional $4 \mathrm{~h}$. Dimethyl sulfoxide was added into each well to dissolve the formazan particles. The absorbance was measured at $450 \mathrm{~nm}$ using a microplate reader (Molecular Devices, LLC).

TUNEL assay. BEAS-2B cells treated with PHI (12.5, 25 or $50 \mu \mathrm{g} / \mathrm{ml} ; 24 \mathrm{~h} ; 37^{\circ} \mathrm{C}$ ) and LPS were fixed with $4 \%$ paraformaldehyde at $25^{\circ} \mathrm{C}$ for $15 \mathrm{~min}$. Then, a TUNEL kit (Roche Applied Science) was used to detect apoptotic cells according to the manufacturer's protocol. Subsequently, cell nuclei were counterstained with $0.2 \mu \mathrm{g} / \mathrm{ml}$ DAPI at room temperature for $15 \mathrm{~min}$ and mounted with glycerol gelatin (Sigma-Aldrich; Merck KGaA). An Olympus fluorescence microscope (magnification, x200) was used to acquire the images in $\geq 3$ randomly selected fields of view.

ELISA. BEAS-2B cells were plated in 96-well plates $\left(8 \times 10^{4}\right.$ cells $\left./ \mathrm{ml}\right)$ and incubated with LPS in the presence or absence of PHI for $24 \mathrm{~h}$. The concentration levels of IL-1 $\beta$, IL- 6 and TNF- $\alpha$ in the cell supernatant were measured by ELISA kits according to the manufacturer's recommendations. The following ELISA kits were purchased from Beyotime Institute of Biotechnology, IL-1 $\beta$ ELISA kit (cat. no. PI305), IL-6 ELISA kit (cat. no. PI330) and TNF- $\alpha$ ELISA kit (cat. no. PT518). The absorbance at $450 \mathrm{~nm}$ was detected by a microplate reader.

$R T-q P C R$. Total RNA in the cells and the serum of pediatric patients with ALI was extracted using TRIzol ${ }^{\circledR}$ reagent (Invitrogen; Thermo Fisher Scientific, Inc.) in accordance with the manufacturer's protocol. Subsequently, the extracts were dissolved and the final RNA purity was measured by the Nucleic Acid/Protein Analyzer (Invitrogen; Thermo Fisher Scientific, Inc.). cDNA was synthesized using 5X All-In-One Master Mix (Invitrogen; Thermo Fisher Scientific, Inc.) in accordance with the manufacturer's protocols. RT-qPCR reactions were performed using SYBR-Green Master Mix (Invitrogen; Thermo Fisher Scientific, Inc.). The reaction conditions were as follows: Initial denaturation at $95^{\circ} \mathrm{C}$ for $10 \mathrm{~min}$, followed by 40 cycles at $95^{\circ} \mathrm{C}$ for $15 \mathrm{sec}$ and $60^{\circ} \mathrm{C}$ for $30 \mathrm{sec}$. The mRNA levels of the target genes were normalized to the levels of the GAPDH gene. The $2^{-\Delta \Delta \mathrm{Cq}}$ (17) method was used for analysis of the data. The sequence of primer pairs were as follows: MMP8 forward (F), 5'-AAGCCATTGATG CAGCTGTTT-3' and reverse (R), 5'-AAACAGCTGCATCAA TGGCTT-3'; and GAPDH F, 5'-CTGAGTACGTCGTGGAGT C-3' and R, 5'-TGATGATCTTGAGGCTGTTGTC-3'. 
A<smiles>COc1cc(C2OCC3COCC32)ccc1O</smiles>

D

G
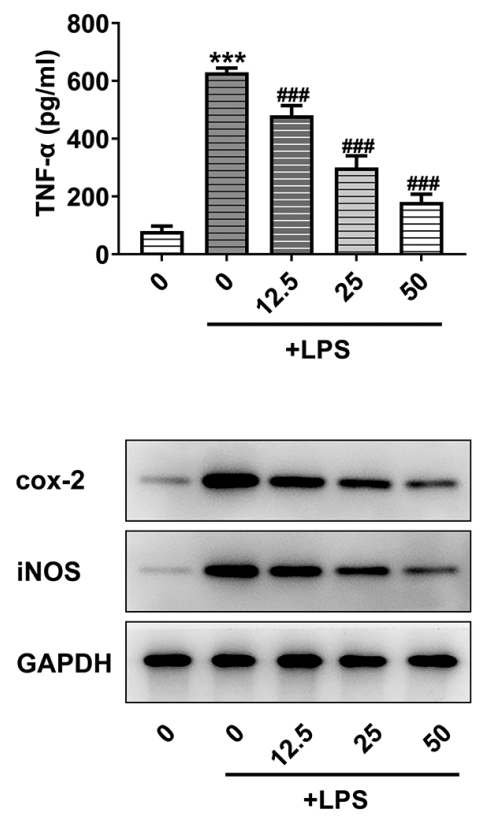

B

E
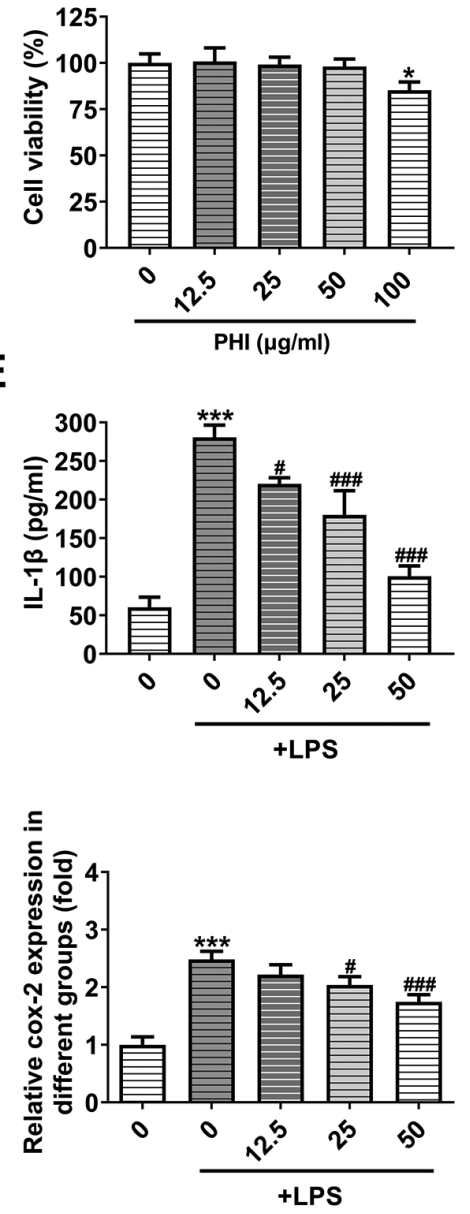

C

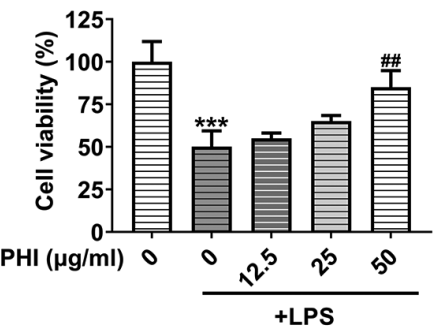

F
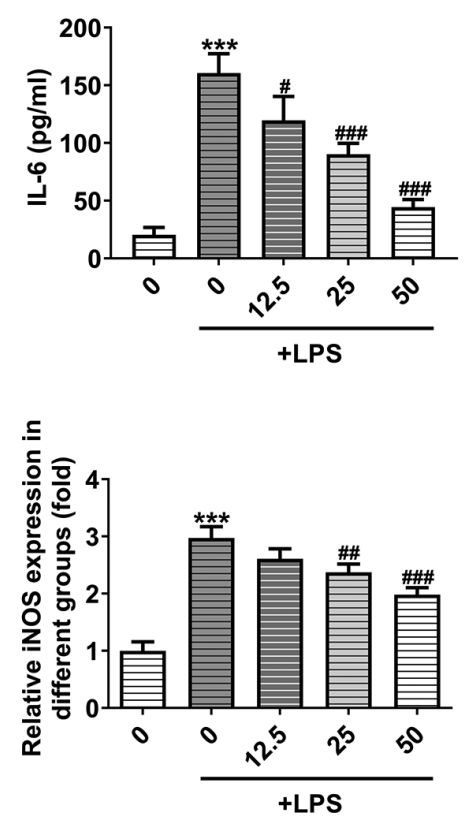

Figure 1. PHI treatment suppresses the inflammatory response in LPS-induced BEAS-2B cells. (A) The chemical structure of PHI. (B) The effects of PHI on the viability of BEAS-2B cells were detected via an MTT assay. ${ }^{*} \mathrm{P}<0.05$ vs. control. (C) The effects of PHI on the viability of BEAS-2B cells induced by LPS were detected via an MTT assay. ${ }^{* * *} \mathrm{P}<0.001$ vs. control; ${ }^{\# \#} \mathrm{P}<0.01$ vs. LPS group. (D-F) The expression levels of inflammatory factors were measured by ELISA. ${ }^{* * *} \mathrm{P}<0.001$ vs. control; ${ }^{\#} \mathrm{P}<0.05,{ }^{\# \# \#} \mathrm{P}<0.001$ vs. LPS group. $(\mathrm{G})$ The levels of inflammation-associated proteins were detected via western blotting. ${ }^{* * * *} \mathrm{P}<0.001$ vs. control; ${ }^{\#} \mathrm{P}<0.05,{ }^{\# \#} \mathrm{P}<0.01,{ }^{\# \#} \mathrm{P}<0.001$ vs. LPS group. PHI, phillygenin; LPS, lipopolysaccharide; cox-2, cyclooxygenase-2; iNOS, inducible nitric oxide synthase.

Western blot analysis. BEAS-2B cells were lysed in RIPA lysis buffer (Beyotime Institute of Biotechnology) and the protein content was estimated using a BCA assay. The samples $(30 \mu \mathrm{g})$ were subsequently loaded on SDS-PAGE gels (10, 12 or 15\%), which were prepared previously. The gels were then transferred to nitrocellulose membranes. The membranes were first blocked using fat-free milk (5\%) in TBS for $2 \mathrm{~h}$ at room temperature and then the membranes were incubated at $4^{\circ} \mathrm{C}$ for $24 \mathrm{~h}$ with primary antibodies against the following: Cox-2 (1:1,000; cat. no. sc-376861), Bcl-2 (1:1,000; cat. no. sc-7382), Bax (1:1,000; cat. no. sc-7480), caspase3 (1:1,000; cat. no. sc-7272), caspase9 (1:1,000; cat. no. sc-56076), MMP8 (1:1,000; cat. no. sc-137044), PPAR $\gamma(1: 1,000$; cat. no. sc-7273), GAPDH (1:2,000; cat. no. sc-47724; all purchased from Santa Cruz Biotechnology, Inc.), iNOS (1:1,000; cat. no. 13120), cleaved (c)-caspase3 (1:1,000; cat. no. 9661) and c-caspase9 (1:1,000; cat. no. 9509; all purchased from Cell Signaling Technology, Inc.). Following which, the membranes were incubated with HRP-conjugated secondary antibody (1:1,000; cat. nos. 7074 and 7076; Cell Signaling Technology, Inc.) for $50 \mathrm{~min}$ at $25^{\circ} \mathrm{C}$.

TBS with Tween-20 (0.2\%) was used to wash the membranes to remove non-specific binding of the antibodies and the ECL luminescence agent (Santa Cruz Biotechnology, Inc.) was added. The images were captured in a Bio-Rad chemiluminescence imager (Bio-Rad Laboratories, Inc.). The expression levels of the proteins were analyzed using ImageJ software version 1.4 (National Institutes of Health) and normalized to those of the control.

Statistical analysis. Data analysis was performed with SPSS version 23.0 (IBM Corp.) and GraphPad (version 5.0; GraphPad Software, Inc.). All experiments were repeated three times and the data are expressed as the mean \pm standard deviation. Two group comparisons were performed using an unpaired Student's t-test was used for the comparison between two group of samples and statistical differences between groups were analyzed using one-way ANOVA followed by a Tukey's post hoc test. $\mathrm{P}<0.05$ was considered to indicate a statistically significant difference.

\section{Results}

PHI treatment suppresses the inflammatory response in $L P S$-induced BEAS-2B cells. To determine the effects of PHI on 
A

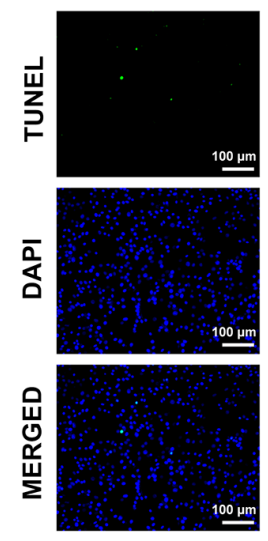

0
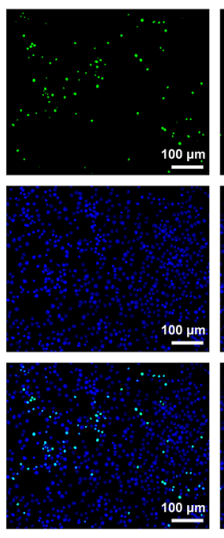

0
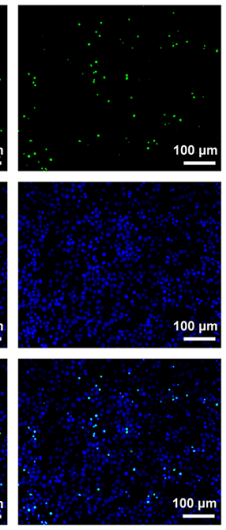

$\imath^{5}$
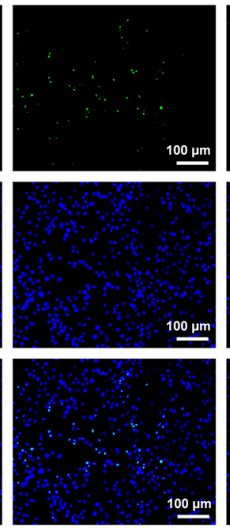

$v^{5}$
B

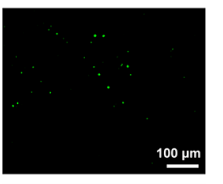

${ }_{100 \mathrm{~mm}}$

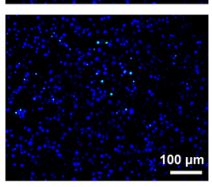

ऽ

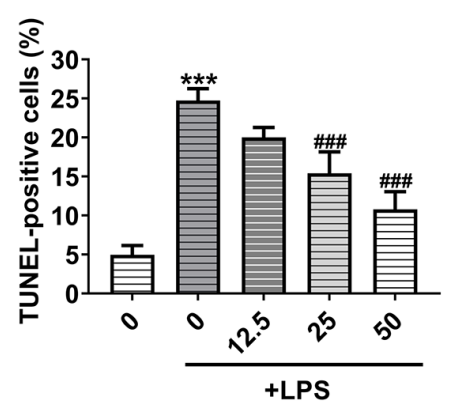

C

+LPS
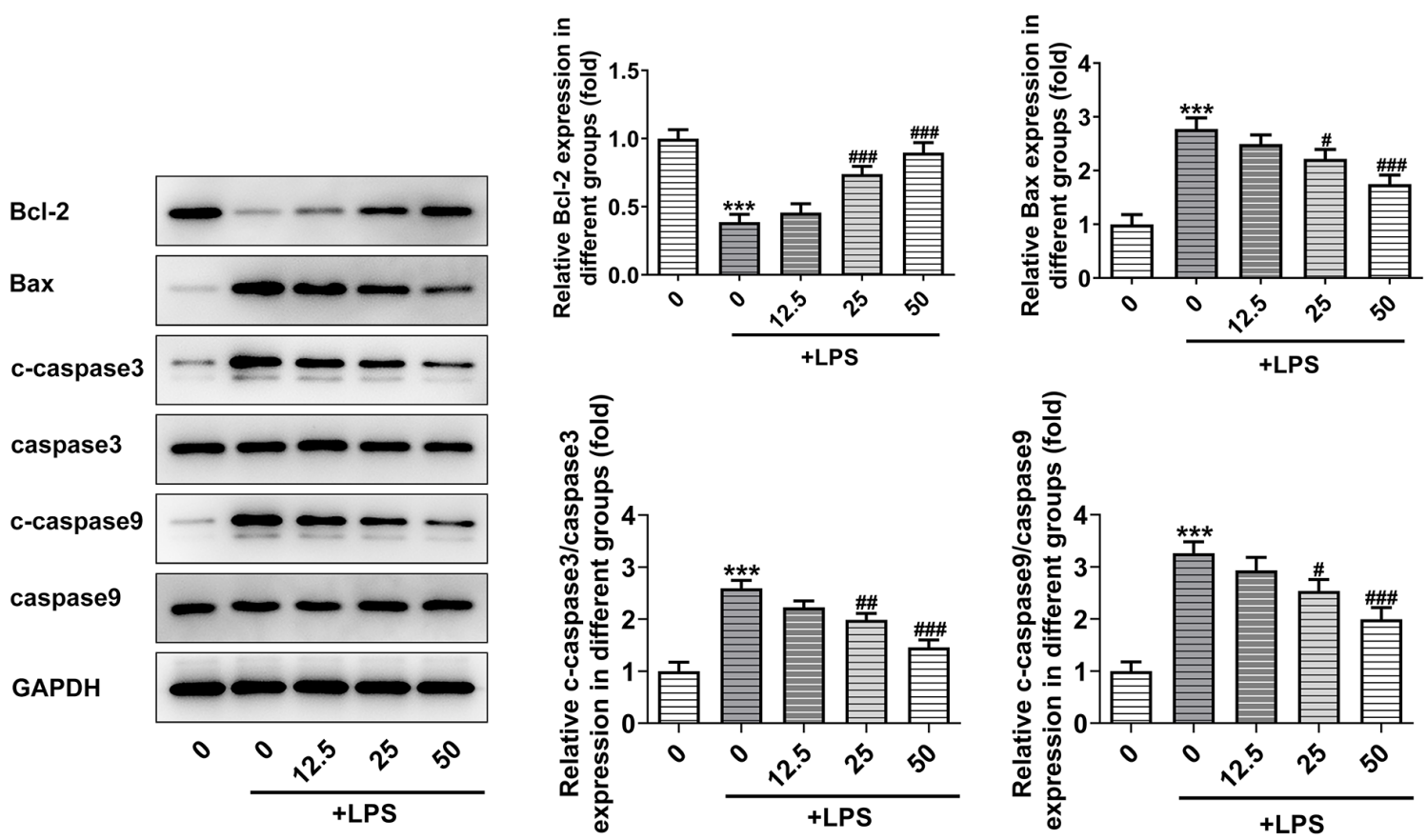

Figure 2. PHI treatment suppresses the induction of apoptosis in BEAS-2B cells. (A and B) Cell apoptosis of LPS-induced BEAS-2B cells was detected using a TUNEL assay (magnification, $\mathrm{x} 200$; scale bar, $100 \mu \mathrm{m}$ ). ${ }^{* * *} \mathrm{P}<0.001$ vs. control; ${ }^{* \# \prime} \mathrm{P}<0.001$ vs. LPS group. (C) Western blotting was used for the analysis of the expression levels of the apoptosis-associated proteins. ${ }^{* * *} \mathrm{P}<0.001$ vs. control; ${ }^{\#} \mathrm{P}<0.05,{ }^{\# \#} \mathrm{P}<0.01,{ }^{\# \# \# /} \mathrm{P}<0.001$ vs. LPS group. PHI, phillygenin; LPS, lipopolysaccharide; c-, cleaved.

pulmonary epithelial cells, BEAS-2B cell viability was detected. Treatment of BEAS-2B cells with PHI $(12.5,25$ and $50 \mu \mathrm{g} / \mathrm{ml})$ indicated no apparent difference in cell viability, while significant differences were noted at $100 \mu \mathrm{g} / \mathrm{ml}$ PHI treatment (Fig. 1B). The cell viability of BEAS-2B cells treated with LPS was markedly decreased. This effect was partially reversed by PHI treatment at 12.5, 25 and $50 \mu \mathrm{g} / \mathrm{ml}$ (Fig. 1C). Therefore, the following concentrations of PHI were selected for subsequent studies: 12.5, 25 and $50 \mu \mathrm{g} / \mathrm{ml}$. ELISA and western blot analysis indicated that the levels of inflammatory cytokines and inflammation-associated proteins were elevated following LPS induction, which could be gradually reversed by PHI treatment at increasing concentrations (Fig. 1D-G). Therefore, these results indicated that PHI treatment suppressed the inflammatory response in LPS-induced BEAS-2B cells.
PHI treatment suppresses the induction of BEAS-2B cell apoptosis. Induction of cell apoptosis plays a critical role in the development of ALI (18). The apoptotic effects of PHI on BEAS-2B cells were confirmed by TUNEL assay and western blot analysis. As shown in Fig. 2A and B, the induction of cell apoptosis was increased by LPS, while increasing doses of PHI alleviated the effect of LPS on BEAS-2B cell apoptosis. In addition, the expression levels of the pro-apoptotic proteins were increased by LPS, while they were decreased by PHI. The opposite finding was noted for the anti-apoptotic protein Bcl-2, which demonstrated decreased expression following treatment with LPS and increased expression by PHI in a dose-dependent manner (Fig. 2C). Taken together, the data revealed that PHI treatment suppressed the induction of BEAS-2B cell apoptosis. 
A

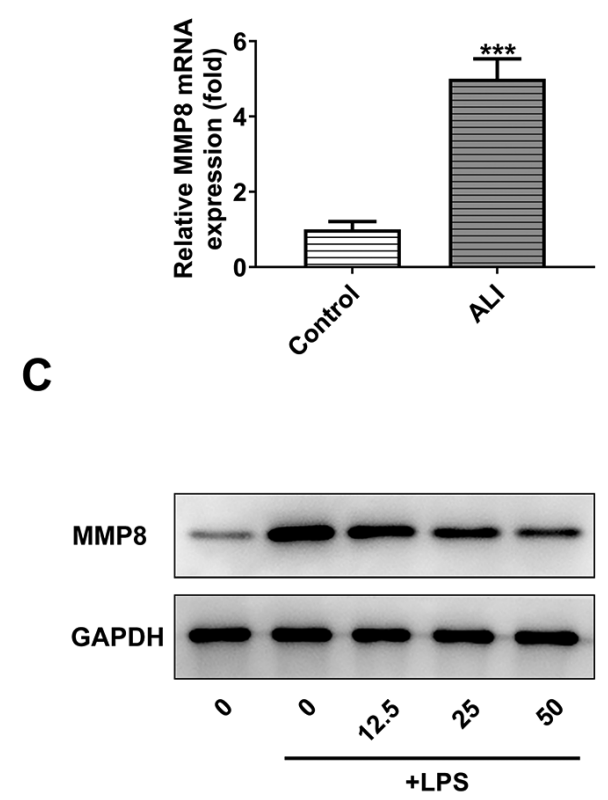

B
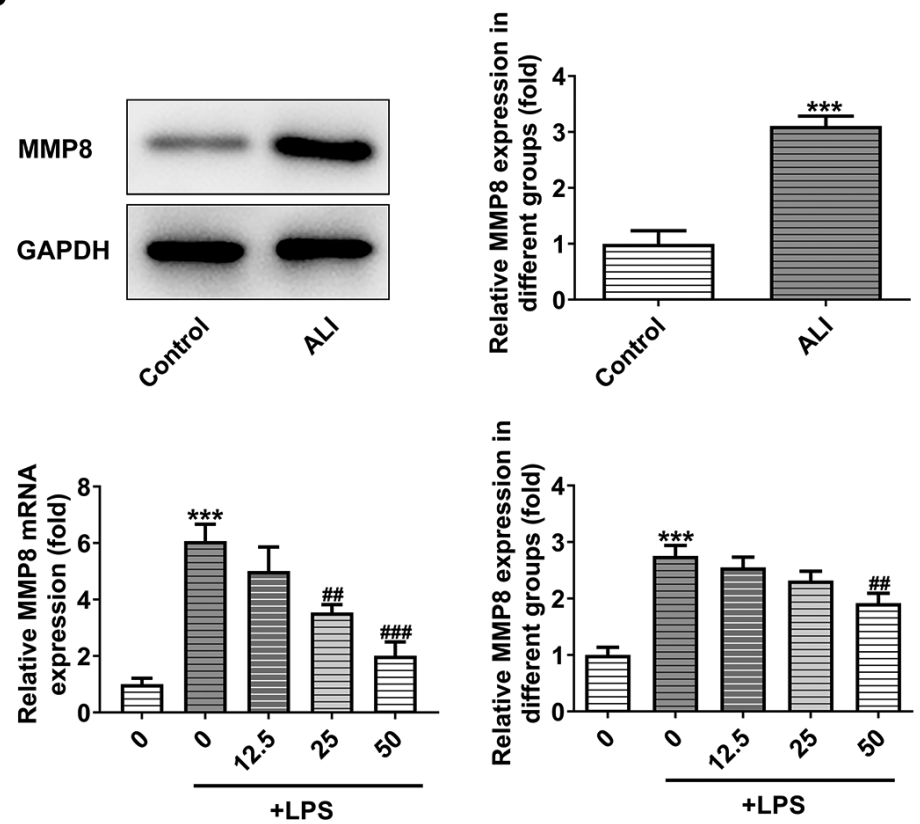

Figure 3. PHI treatment decreases the expression levels of MMP8 in LPS-induced BEAS-2B cells. (A) The mRNA and (B) protein levels of MMP8 were detected via reverse transcription-quantitative PCR and western blot analyses, respectively. ${ }^{*}{ }^{* *} \mathrm{P}<0.001$ vs. control. (C) The expression levels of MMP8 in LPS-induced BEAS-2B cells following PHI treatment were detected via western blotting. ${ }^{* * *} \mathrm{P}<0.001$ vs. control; ${ }^{\# \#} \mathrm{P}<0.01,{ }^{\# \#} \mathrm{P}<0.001$ vs. LPS group. PHI, phillygenin; LPS, lipopolysaccharide; ALI, acute lung injury.

PHI treatment decreases the expression levels of MMP8 in LPS-induced BEAS-2B cells. Serum samples of normal subjects and pediatric patients with ALI were collected and assessed by RT-qPCR and western blot analyses. As demonstrated in Fig. 3A and B, the expression levels of MMP8 were significantly increased in patients with ALI. Following the addition of increasing doses of PHI in LPS-induced BEAS-2B cells, western blot analysis demonstrated that the expression levels of MMP8 were gradually decreased (Fig. 3C). Therefore, PHI treatment decreased the expression levels of MMP8 in LPS-induced BEAS-2B cells.

PHI treatment suppresses induction of inflammation in LPS-treated BEAS-2B cells by downregulating MMP8 expression. To directly assess the effects of MMP8 on LPS-induced BEAS-2B cells, which were treated with PHI, $50 \mu \mathrm{g} / \mathrm{ml}$ PHI was selected for subsequent experiments and MMP8-overexpression models were established. RT-qPCR and western blot analyses indicated significantly higher levels of MMP8 in the pcDNA3.1-MMP8 group compared with those of the control group (Fig. 4A and B). The expression levels of the inflammatory factors, which were inhibited by PHI in LPS-induced BEAS-2B cells, were significantly elevated following transfection of pcDNA3.1-MMP8 into the cells (Fig. 4C-E). Moreover, the inflammation-associated proteins exhibited decreased expression following PHI treatment in LPS-induced BEAS-2B cells, which could be partially reversed by MMP8 overexpression (Fig. 4F). These results indicated that PHI treatment suppressed the inflammation of LPS-induced BEAS-2B cells.

PHI treatment activates PPAR $\gamma$ signaling by downregulating MMP8 expression. The induction of apoptosis of LPS-treated
BEAS-2B cells was detected by TUNEL staining. PHI decreased the number of apoptotic cells in LPS-induced BEAS-2B cells, while pcDNA3.1-MMP8 increased it, indicating that PHI treatment suppressed the induction of apoptosis in LPS-treated BEAS-2B cells (Fig. 5A and B). Moreover, the changes in the protein levels of the anti-apoptotic and pro-apoptotic proteins further demonstrated that MMP8 overexpression partially reduced the inhibitory effects of PHI on cell apoptosis (Fig. 5C). The results from the western blot analysis indicated that PPAR $\gamma$ expression was significantly decreased following LPS treatment of the cells, while additional PHI treatment rescued its expression (Fig. 5D). This change was partially reversed by MMP8 overexpression (Fig. 5D). Therefore, these results suggested that PHI treatment activated PPAR $\gamma$ signaling by downregulating MMP8 expression.

\section{Discussion}

Forsythiae Fructus is used as a single herb or added in compound prescriptions in Asia (19). Dong et al (20) was the first to report the use of this type of dried fruit as lianqiao (Forsythia). It has been used for the treatment of infectious diseases, including acute nephritis, since it exhibits heat-clearing and detoxification effects (21). The Forsythia Fructus extract further exerts anti-inflammatory, antibacterial, antiviral and antioxidant effects $(22,23)$. PHI is the extract from Forsythia Fructus, which demonstrates antitumor, antioxidant and hepatoprotective effects in vivo and in vitro $(24,25)$. A previous study has observed the anti-inflammatory activity of PHI in mouse lymphocytes (9). Moreover, Forsythia Fructus could enhance the defense mechanism in rats with LPS-induced liver injury (26). Concurrently, the present study indicated the importance of 
A

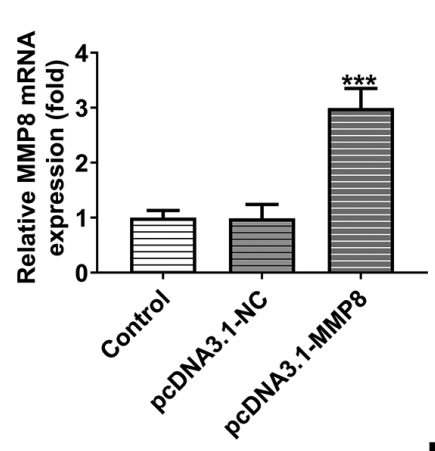

C

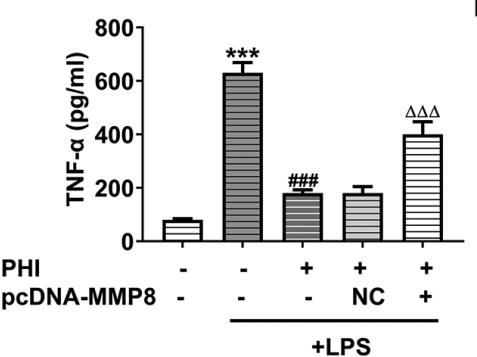

$\mathbf{F}$

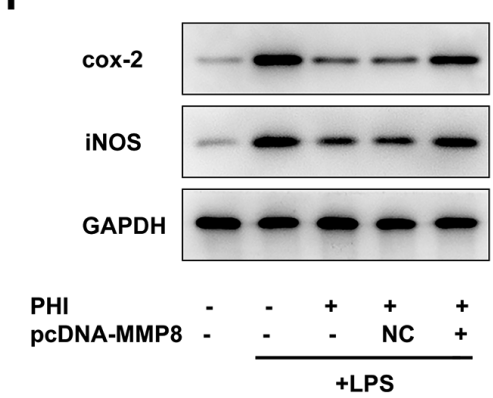

B

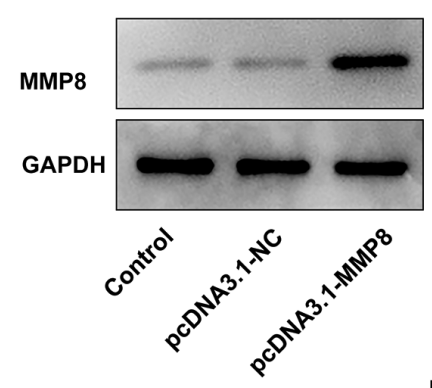

D

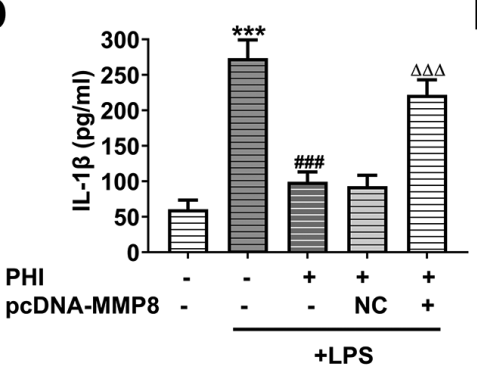

E
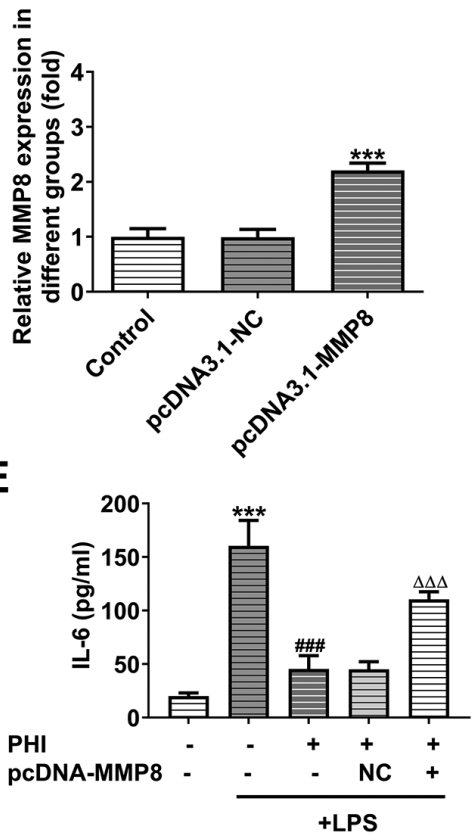

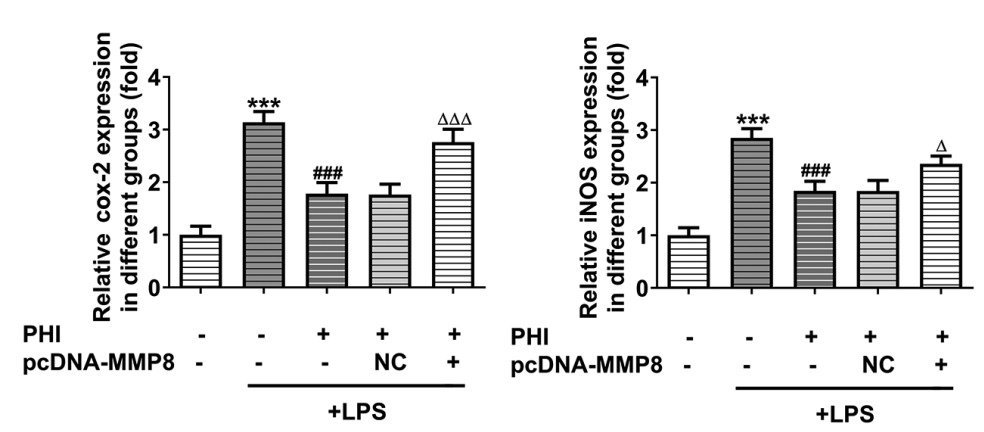

Figure 4. PHI treatment suppresses inflammation of LPS-induced BEAS-2B cells. pcDNA3.1-MMP8 was constructed and the expression levels of MMP8 were measured via (A) reverse transcription-quantitative PCR and (B) western blot analyses. ${ }^{* * *} \mathrm{P}<0.001$ vs. control and pcDNA3.1-NC. (C-E) Following transfection of pcDNA3.1-MMP8 into LPS-induced BEAS-2B cells pretreated with PHI, the expression levels of the inflammatory factors were measured via ELISA. ${ }^{* * * *} \mathrm{P}<0.001$ vs. control; ${ }^{\# \#} \mathrm{P}<0.001$ vs. LPS group; ${ }^{\Delta \Delta \Delta} \mathrm{P}<0.001$ vs. LPS + PHI group. (F) Following transfection of pcDNA3.1-MMP8 into LPS-induced BEAS-2B cells pretreated with PHI, the expression levels of the inflammation-associated factors were measured via western blotting. ${ }^{* * *} \mathrm{P}<0.001$ vs. control;

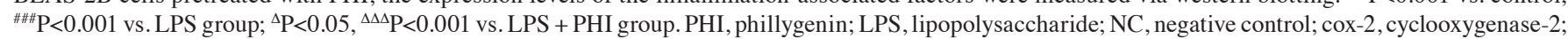
iNOS, inducible nitric oxide synthase.

PHI in alleviating inflammation and reducing apoptosis in LPS-induced BEAS-2B cells.

The generation of ROS and the reduction in MMP expression levels have been reported to be important events in triggering apoptosis. PHI was previously found to increase ROS and decrease MMP levels in promyelocytic leukemia HL-60 cells, indicating its effects on the suppression of cell apoptosis (27). A previous study reported the effects of MMP8 deletion on the improvement of septic patient survival and the suppression of the inflammatory response in a murine model (28). MMP8 inhibitors have also been considered key regulators in mitigating myocardial injury (29). In the present study, MMP8 was found to be highly expressed in the serum of pediatric patients with ALI, whereas PHI treatment decreased the levels of MMP8 expression. Further experiments confirmed the regulatory role of PHI in the expression of MMP8, and it was concluded that PHI alleviated inflammation and reduced apoptosis in LPS-induced BEAS-2B cells by downregulating MMP8 expression levels.
PPARs are ligand-activated transcription factors that exert anti-inflammatory effects on certain diseases, including brain injury $(30,31)$. Furthermore, PPARs also regulate the transcription of genes with critical roles in various cellular processes $(32,33)$. These transcription factors belong to the superfamily of nuclear receptors and they are present in a variety of tissues for cellular homeostasis. As PPARs are involved in the development of numerous diseases, such as brain and peripheral inflammation and cancer, and they are considered to be potential molecular targets $(30,34,35)$. Previously, it was shown that PPAR $\gamma$ plays an important role in the regulation of gene expression, such as that of lipoprotein(a), IL-1 and TNF- $\alpha(36,37)$. The activation of PPAR $\gamma$ can also regulate different cellular processes, including cell proliferation, metabolism and inflammation (38). In the present study, PPAR $\gamma$ signaling was inhibited by LPS induction in BEAS-2B cells, whereas PHI treatment led to an increase in the expression of PPAR $\gamma$ in LPS-induced BEAS-2B cells. However, MMP8 overexpression partially alleviated the effects of PHI on activating the PPAR $\gamma$ signaling pathway. 
A

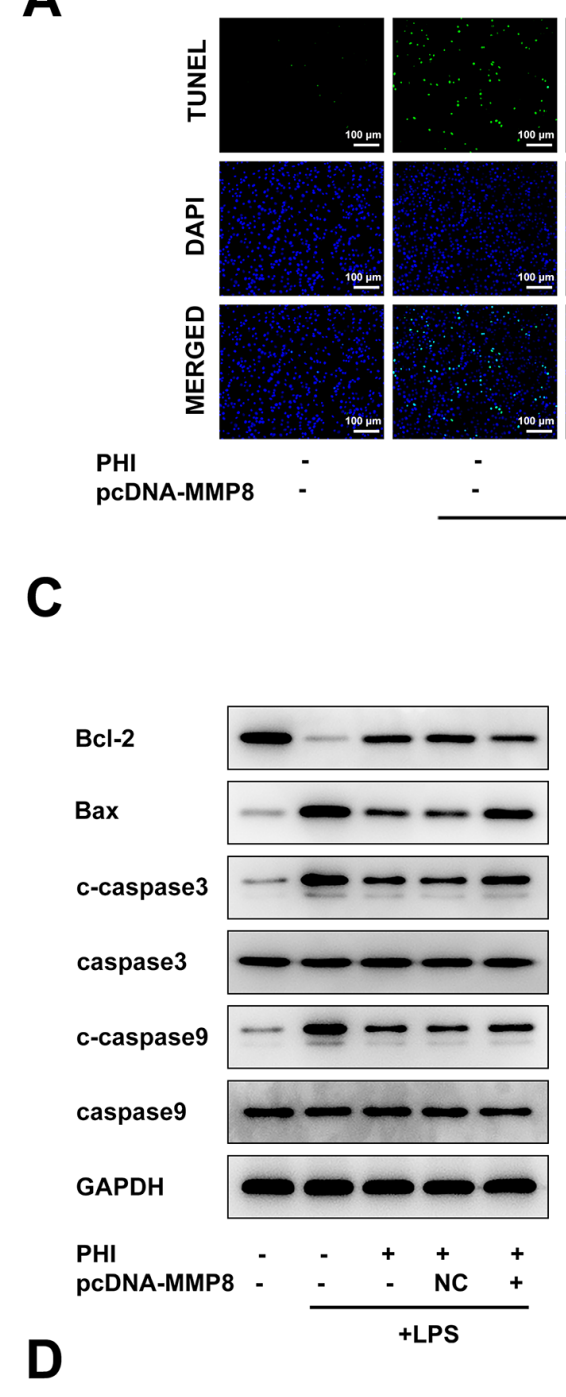

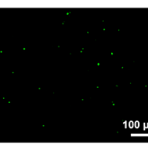
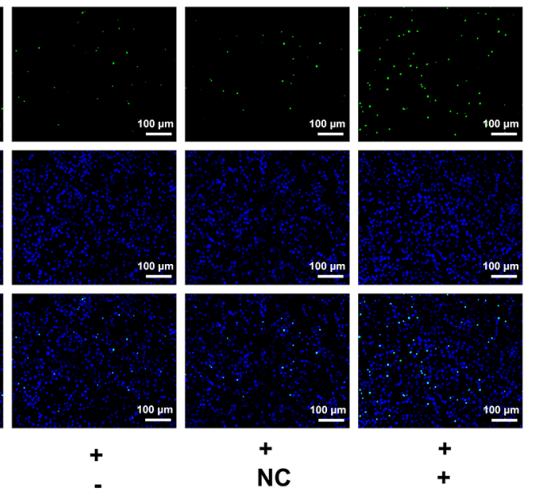

B

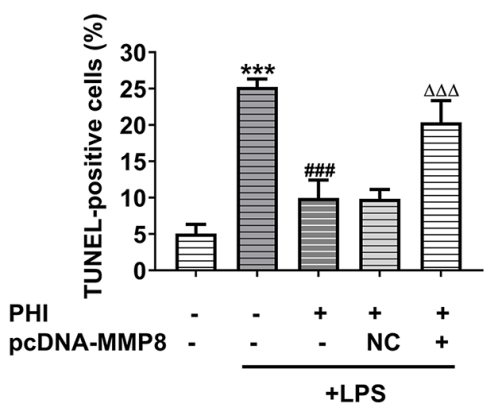

+LPS
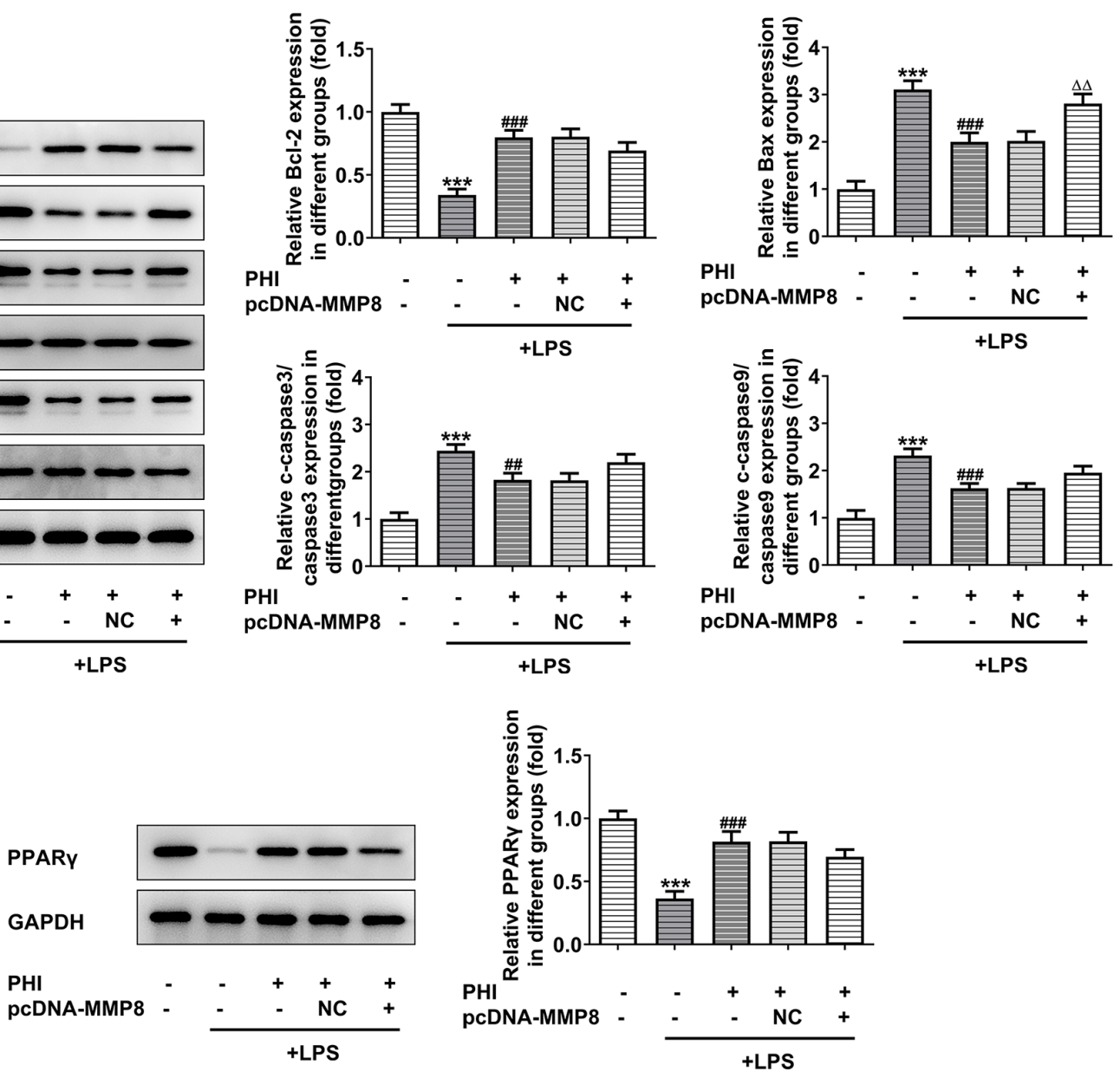

Figure 5. PHI treatment activates PPAR $\gamma$ signaling by downregulating MMP8 expression. (A and B) Following MMP8 overexpression, the induction of cell apoptosis of LPS-treated BEAS-2B cells, which were pretreated with PHI, was assessed using a TUNEL assay (magnification, 200 ; scale bar, $100 \mu$ m). ${ }^{* * *} \mathrm{P}<0.001$ vs. control; ${ }^{\# \# \#} \mathrm{P}<0.001$ vs. LPS group; ${ }^{\Delta \Delta \Delta} \mathrm{P}<0.001$ vs. LPS + PHI group. (C) Western blotting was used to determine the expression levels of the apoptosis-associated proteins in LPS-induced BEAS-2B cells treated with PHI and transfected with pcDNA3.1-MMP8. *** $<<0.001$ vs. control; \# ${ }^{*}<0.01$,

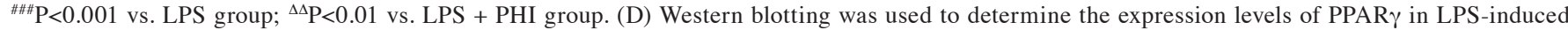
BEAS-2B cells treated with PHI and transfected with pcDNA3.1-MMP8. ${ }^{* * *}$ P $<0.001$ vs. control; ${ }^{\# \# P<0.001 ~ v s . ~ L P S ~ g r o u p . ~ P H I, ~ p h i l l y g e n i n ; ~ P P A R ~} \gamma$, peroxisome proliferator-activated receptor $\gamma$; LPS, lipopolysaccharide; NC, negative control; c-, cleaved.

In conclusion, the present study demonstrated that PHI inhibited inflammation and apoptosis of pulmonary epithelial cells by activating PPAR $\gamma$ signaling via downregulation of MMP8 expression, which may guide future studies on PHI and provide a theoretical basis for the therapeutic potential of PHI. However, there are numerous limitations of the present study. After validating the effect of PHI on LPS-induced inflammation and apoptosis in lung epithelial cells in vitro, it would improve the results of the present study if experiments and comparison tests with positive control tests had been performed in animal models.

\section{Acknowledgements}

Not applicable. 


\section{Funding}

No funding was received.

\section{Availability of data and materials}

The datasets used and/or analyzed during the current study are available from the corresponding author on reasonable request.

\section{Authors' contributions}

PY and YL collaborated on the manuscript, including designing the research, collecting and analyzing the experiments and data, and writing the manuscript. PY and YL confirmed the authenticity of all the raw data. Both authors read and approved the final manuscript.

\section{Ethics approval and consent to participate}

The clinical study protocol was approved by Gaolangang Hospital of Zhuhai People's Hospital (Zhuhai, China; approval no. 2020-012). Signed informed consent forms were obtained from the children's guardians for the collection and use of the specimens.

\section{Patient consent for participation}

Not applicable.

\section{Competing interests}

The authors declare that they have no competing interests.

\section{References}

1. Moreira Lopes TC, Mosser DM and Gonçalves R: Macrophage polarization in intestinal inflammation and gut homeostasis. Inflamm Res 69: 1163-1172, 2020.

2. Miyajima A: Cytokines and their functions. Nihon Rinsho 63 (Suppl 4): S173-S177, 2005 (In Japanese)

3. Fidalgo P, Ahmed M, Meyer SR, Lien D, Weinkauf J, Cardoso FS, Jackson $\mathrm{K}$ and Bagshaw SM: Incidence and outcomes of acute kidney injury following orthotopic lung transplantation: A population-based cohort study. Nephrol Dial Transplant 29: 1702-1709, 2014

4. Jin W and Dong C: IL-17 cytokines in immunity and inflammation. Emerg Microbes Infect 2: e60, 2013.

5. Tomashefski JF Jr: Pulmonary pathology of acute respiratory distress syndrome. Clin Chest Med 21: 435-466, 2000.

6. Chen H, Bai $C$ and Wang $X$ : The value of the lipopolysaccharide-induced acute lung injury model in respiratory medicine. Expert Rev Respir Med 4: 773-783, 2010.

7. Li Y, Huang J, Foley NM, Xu Y, Li YP, Pan J, Redmond HP, Wang JH and Wang J: B7H3 ameliorates LPS-induced acute lung injury via attenuation of neutrophil migration and infiltration. Sci Rep 6: 31284, 2016.

8. Hu N, Wang C, Dai X, Zhou M, Gong L, Yu L, Peng C and Li Y: Phillygenin inhibits LPS-induced activation and inflammation of LX2 cells by TLR4/MyD88/NF- $\kappa$ B signaling pathway. J Ethnopharmacol 248: 112361, 2020.

9. Du B, Zhang L, Sun Y, Zhang G, Yao J, Jiang M, Pan L and Sun C: Phillygenin exhibits anti-inflammatory activity through modulating multiple cellular behaviors of mouse lymphocytes. Immunopharmacol Immunotoxicol 41: 76-85, 2019.

10. Li H, Chen M, Yang Z, Wang Q, Wang J, Jin D, Yang X, Chen F, Zhou X and Luo K: Phillygenin, a MELK Inhibitor, inhibits cell survival and Epithelial-Mesenchymal transition in pancreatic cancer cells. Onco Targets Ther 13: 2833-2842, 2020.
11. He J, Wei W, Yang Q and Wang Y: Phillygenin exerts in vitro and in vivo antitumor effects in drug-resistant human esophageal cancer cells by inducing mitochondrial-mediated apoptosis, ROS generation, and inhibition of the nuclear factor kappa B NF-kappaB signalling pathway. Med Sci Monit 25: 739-745, 2019.

12. Zinter MS, Delucchi KL, Kong MY, Orwoll BE, Spicer AS, Lim MJ, Alkhouli MF, Ratiu AE, McKenzie AV, McQuillen PS, et al: Early plasma matrix metalloproteinase profiles. A novel pathway in pediatric acute respiratory distress syndrome. Am J Respir Crit Care Med 199: 181-189, 2019.

13. Kong MY, Gaggar A, Li Y, Winkler M, Blalock JE and Clancy JP: Matrix metalloproteinase activity in pediatric acute lung injury. Int J Med Sci 6: 9-17, 2009.

14. Kumar H, Jo MJ, Choi H, Muttigi MS, Shon S, Kim BJ, Lee SH and Han IB: Matrix metalloproteinase- 8 inhibition prevents disruption of blood-spinal cord barrier and attenuates inflammation in rat model of spinal cord injury. Mol Neurobiol 55: 2577-2590, 2018.

15. Lee EJ, Park JS, Lee YY, Kim DY, Kang JL and Kim HS: Anti-inflammatory and anti-oxidant mechanisms of an MMP-8 inhibitor in lipoteichoic acid-stimulated rat primary astrocytes: Involvement of NF- $\kappa \mathrm{B}, \mathrm{Nrf} 2$, and PPAR $-\gamma$ signaling pathways. J Neuroinflammation 15: 326, 2018.

16. Cheng K, Yang A, Hu X, Zhu D and Liu K: Curcumin attenuates pulmonary inflammation in lipopolysaccharide induced acute lung injury in neonatal rat model by activating peroxisome proliferator-activated receptor $\gamma$ (PPAR $\gamma$ ) Pathway. Med Sci Monit 24: 1178-1184, 2018.

17. Livak KJ and Schmittgen TD: Analysis of relative gene expression data using real-time quantitative PCR and the 2(-Delta Delta C(T)) method. Methods 25: 402-408, 2001.

18. Chen C, Zhang Z, Tan F, Meng F, Lai L, Chi X and Zhu Q: Stabilizing mast cells improves acute lung injury after orthotopic liver transplantation via promotion of apoptosis in polymorphonuclear neutrophils. Am J Physiol Lung Cell Mol Physiol 320: L266-L275, 2021.

19. Zhou M, Yu S, Hong B, Li J, Han H and Qie G: Antibiotics control in aquaculture requires more than antibiotic-free feeds: A tilapia farming case. Environ Pollut 268: 115854, 2021.

20. Dong Z, Lu X, Tong X, Dong Y, Tang L and Liu M: Forsythiae fructus: A review on its phytochemistry, quality control, pharmacology and pharmacokinetics. Molecules 22: 1466, 2017.

21. Jia J, Zhang F, Li Z, Qin X and Zhang L: Comparison of fruits of forsythia suspensa at two different maturation stages by NMR-based metabolomics. Molecules 20: 10065-10081, 2015.

22. Cao J, Shao SY, Zhang X, Yuan X, Feng ZM, Jiang JS, Yang YN and Zhang PC: Two new lignans from the fruits of Forsythia suspensa. J Asian Nat Prod Res 22: 418-424, 2020.

23. Ko HC, Wei BL and Chiou WF: Dual regulatory effect of plant extracts of Forsythia suspense on RANTES and MCP-1 secretion in influenza A virus-infected human bronchial epithelial cells. J Ethnopharmacol 102: 418-423, 2005.

24. Guo N, Gai QY, Jiao J, Wang W, Zu YG and Fu YJ: Antibacterial activity of fructus forsythia essential oil and the application of EO-Loaded nanoparticles to food-borne pathogens. Foods 5: 73, 2016.

25. Song W, Wu J, Yu L and Peng Z: Evaluation of the pharmacokinetics and hepatoprotective effects of phillygenin in mouse. Biomed Res Int 2018: 7964318, 2018.

26. Hao Y, Li D, Piao X and Piao X: Forsythia suspensa extract alleviates hypersensitivity induced by soybean beta-conglycinin in weaned piglets. J Ethnopharmacol 128: 412-418, 2010.

27. Duan D, Zhang B, Yao J, Liu Y and Fang J: Shikonin targets cytosolic thioredoxin reductase to induce ROS-mediated apoptosis in human promyelocytic leukemia HL-60 cells. Free Radic Biol Med 70: 182-193, 2014.

28. Solan PD, Dunsmore KE, Denenberg AG, Odoms K, Zingarelli B and Wong HR: A novel role for matrix metalloproteinase- 8 in sepsis. Crit Care Med 40: 379-387, 2012.

29. Zhou X, Lu J, Chen D, Wang W, Cai Q, Li T and Zhang J: Matrix metalloproteinase- 8 inhibitors mitigate sepsis-induced myocardial injury in rats. Chin Med J (Engl) 127: 1530-1535, 2014.

30. Villapol S: Roles of peroxisome proliferator-activated receptor gamma on brain and peripheral inflammation. Cell Mol Neurobiol 38: 121-132, 2018.

31. Ju Z, Su M, Hong J, Kim E and Jung JH: Anti-inflammatory effects of an optimized PPAR- $\gamma$ agonist via NF- $\kappa$ B pathway inhibition. Bioorg Chem 96: 103611, 2020.

32. Yonutas HM and Sullivan PG: Targeting PPAR isoforms following CNS injury. Curr Drug Targets 14: 733-742, 2013. 
33. Kapadia R, Yi JH and Vemuganti R: Mechanisms of anti-inflammatory and neuroprotective actions of PPAR-gamma agonists. Front Biosci 13: 1813-1826, 2008.

34. El Dairi R, Huuskonen P, Pasanen M and Rysä J: Peroxisome proliferator activated receptor gamma (PPAR-gamma) ligand pioglitazone regulated gene networks in term human primary trophoblast cells. Reprod Toxicol 81: 99-107, 2018

35. Yousefnia S, Momenzadeh S, Seyed Forootan F, Ghaedi K and Nasr Esfahani MH: The influence of peroxisome proliferator-activated receptor $\gamma(\operatorname{PPAR} \gamma)$ ligands on cancer cell tumorigenicity. Gene 649: 14-22, 2018.

36. Janani $C$ and Ranjitha Kumari BD: PPAR gamma gene-a review. Diabetes Metab Syndr 9: 46-50, 2015.
37. Hashemzadeh AA, Nasoohi N, Raygan F, Aghadavod E, Akbari E, Taghizadeh M, Memarzadeh MR and Asemi Z: Flaxseed Oil Supplementation improve gene expression levels of PPAR- $\gamma$, LP(a), IL-1 and TNF- $\alpha$ in type 2 diabetic patients with coronary heart disease. Lipids 52: 907-915, 2017.

38. Gurley C, Nichols J, Liu S, Phulwani NK, Esen N and Kielian T: Microglia and astrocyte activation by toll-like receptor ligands: Modulation by PPAR-gamma Agonists. PPAR Res 2008: 453120, 2008.

This work is licensed under a Creative Commons

Attribution-NonCommercial-NoDerivatives 4.0 International (CC BY-NC-ND 4.0) License. 